\title{
Inference With Cross-Lagged Effects - Problems in Time
}

\author{
Charles C. Driver \\ Institute of Education, University of Zurich \\ Institute for Educational Evaluation, Affiliated Institute at the University of Zurich \\ Center for Lifespan Psychology, Max Planck Institute for Human Development
}

\begin{abstract}
The interpretation of cross-effects from vector autoregressive models to infer structure and causality amongst constructs is widespread and sometimes problematic. I describe problems in the interpretation of cross-effects when processes that are thought to fluctuate continuously in time are, as is typically done, modeled as changing only in discrete steps (as in e.g. structural equation modelling) - zeroes in a discrete-time temporal matrix do not necessarily correspond to zeroe effects in the underlying continuous processes, and vice versa. This has implications for the common case when the presence or absence of cross-effects is used for inference about underlying causal processes. I demonstrate these problems via simulation, and also show that when an underlying set of processes are continuous in time, even relatively few direct causal links can result in much denser temporal effect matrices in discrete-time. I demonstrate one solution to these issues, namely parameterizing the system as a stochastic differential equation and focusing inference on the continuous-time temporal effects. I follow this with some discussion of issues regarding the switch to continuous-time, specifically regularization, appropriate measurement time lag, and model order.
\end{abstract}

Keywords: Continuous time, dynamic model, cross-lagged panel, lagged effects, causal inference, multilevel VAR, dual-change score, network analysis, stochastic differential equation

Predicting the future based on a set of past observations is, in at least some sense, a straightforward problem. We can of course be wrong to varying degrees, but the basic tools of statistics and computation have evolved to the point where standard recipes for the use of past data to predict future data can often give quite reasonable results. In contrast, predicting how the future will be different if we intervene in a specific way, based on the same set of past observations, is, in most cases, far more difficult. Therein lies both the promise and the pain of much of statistical modeling. Discussing mere correlation is easy, causal interpretation under the assumption of having fit the 'true' model is easy, but unfortunately most science tends to be different - causality is of interest and models plus data are only approximations of a more complex reality. In classical experimental settings such as a randomized controlled trial, the statistical model can sometimes be quite simple, and the interpretation seem-

Charles C Driver, Institute of Education, University of Zurich. Thanks to Dr. Manuel Voelkle and Dr. Martin Tomasik for comments and critique. Analysis scripts and generated data are available in the supplementary material. Correspondence concerning this article should be addressed to Charles $\mathrm{C}$ Driver, Institute of Education, University of Zurich, Freiestrasse 36, CH-8032 Zurich. E-mail: charles.driver2@uzh.ch ingly clear - the prime inferential difficulty in such cases is usually mapping understanding of one or more very specific, controlled contexts, to the broader and messier reality we're usually interested in (Dekkers et al., 2010; Rothwell, 2006, details some common concerns). The use of observational data approaches typically aims to reduce some of these generalization issues (i.e. data better reflects the circumstances of interest) (Degtiar \& Rose, 2021), but from this arises what can be thought of as an inverse generalization issue - that of going from 'what is likely to happen to $\mathrm{y}$ when $\mathrm{x}$ changes for any reason' to 'what is likely to happen to $\mathrm{y}$ if $\mathrm{x}$ is directly manipulated'. For a tangible example, when the number of people with umbrellas on the street increases, ice cream sales likely decrease, so umbrella carrying may be a useful predictor of ice-cream sales. However, taking peoples umbrellas from them during a downpour is unlikely to increase peoples desire for ice-cream - umbrella carrying and ice cream sales are influenced by a common cause, the weather.

Considering the ice-cream umbrella system described, a critical feature that could help a naive observer make sense of the causal relations in the system is the relationship of the three variables over time. Changes in umbrella carrying and ice-cream buying will tend to happen after changes in the weather. Moreover, after accounting for the information contained in all past states of the weather, umbrella carrying will (likely!) not contain useful information for predicting 
ice-cream sales. Such ideas of temporal causality were formalized by Wiener (Wiener, 1956), made practical in linear model form by Granger (Granger, 1969), and have since seen non-linear generalizations (for an overview see HlaváčkováSchindler et al., 2007). The general tenets of this approach are that causes happen prior to their effects, and causes contain unique information about future effects.

Within this approach, variables are sometimes considered 'Granger causal' - a term reflecting the idea that in a specific model of causality based on the approach of Granger, the variables appear causally linked, but that additional variables or model changes may alter this. This typical view of Granger causality appears then a very weak notion of causality. A more standard view of causality is found in the counterfactual perspective, wherein if we intervened directly on a causal variable we would expect to see the downstream changes predicted by the model (for some major works see Lewis, 1973; Pearl, 2009; Rubin, 2005; Spirtes et al., 2000). Imagine that we do not include the weather variable in an analysis of our earlier system, then umbrella carrying is useful information to predict future ice-cream sales, since it is related to the weather, and might be considered 'Granger causal' according to common parlance. However if we intervene on umbrella carrying at a given point in time, then of course no specific change in later ice-cream sales is to be expected. Granger (Granger, 1980) did discuss at some length the information necessary to include in an analysis of causality, and this can be broadly understood as ensuring there are no unmeasured confounders (i.e. common causes) (Shojaie \& Fox, 2022). The approach of Granger can be seen as a search for genuine causal effects, but conclusions should always be tempered by one's confidence in the many assumptions of the models.

In the psychological domain, models that are commonly used (often implicitly) in the Granger causality framework include the cross-lagged panel (Campbell, 1963), vector autoregressive (VAR) (Orcutt \& Irwin, 1948), latent change score (McArdle, 2001), as well as many extensions and combinations (e.g., Asparouhov et al., 2018, Bringmann et al., 2017, Epskamp et al., 2018; Gates \& Molenaar, 2012, Zyphur et al., 2020). Ruissen et al. (2021) provide a recent overview contrasting some of the different approaches and software. All of these models contain (or can be re-written to contain, e.g. Voelkle \& Oud, 2015) some form of parameter that can be understood as a cross-lagged effect - the (potentially causal) effect of one variable at an earlier time, on another variable at a later time. The common, first order (lag one) version of a VAR can be written as:

$$
y_{u}=\mathcal{A} y_{u-1}+\mathcal{B}+\mathcal{G} z_{u} \quad z \sim N(0,1)
$$

Where $y$ is a $d$ length vector of process values (possibly but not necessarily data), $u$ is an index of measurement occasion, $\mathcal{A}$ is a matrix of temporal regression coefficients, $\mathcal{B}$ is an intercept, and $\mathcal{G}$ is the effect matrix of the $d$ length system noise vector $z$, where $z$ contains independent and identically distributed deviations with zero mean. Many extensions and variations are in use, non-linear forms may be written more generically as functions, but the issues discussed herein, while broadly applicable, are simplest to understand using the basic form shown.

Cross-lagged effects in longitudinal models have for long offered a tantalizing vision of causal inference in observational data, but their interpretation has for just as long been problematic and controversial, as interpretations often go far beyond what can reasonably be said given the data and modelling. Shojaie and Fox (2022) point out a number of important assumptions of the standard VAR model for causal inference. These core assumptions (quoted verbatim from Shojaie \& Fox, 2022) are:

1. Continuous-valued series: All series are assumed to have continuous-valued observations. However, many interesting data sources-such as social media posts or health states of an individual-are discrete-valued.

2. Linearity: The true data generating process, and correspondingly the causal effects of variables on each other, is assumed to be linear. In reality, many real-world processes are nonlinear.

3. Discrete time: The sampling frequency is assumed to be on a discrete, regular grid matching the true causal time lag. If the data acquisition rate is slower or otherwise irregular, causal effects may not be identifiable. Likewise, the analysis of point processes or other continuous-time processes is precluded.

4. Known lag: The (linear) dependency on a history of lagged observations is assumed to have a known order. Classically, the order was not estimated and was taken to be uniform across all series.

5. Stationarity: The statistics of the process are assumed time invariant, whereas many complex processes have evolving relationships (e.g., brain networks vary by stimuli and user activity varies over time and context).

6. Perfectly observed: The variables need to be observed without measurement errors.

7. Complete system: All relevant variables are assumed to be observed and included in the analysis-i.e., there are no unmeasured confounders. This is a stringent requirement, especially given that early approaches for Granger causality focused on the bivariate case-that is, they did not account for any potential confounders.

All these assumptions, except that of the complete system, can be relaxed to varying degrees by different model extensions. While there are undoubtedly difficulties and scope for improvement with respect to all listed items, in this paper I will focus specifically on the discrete-time assumption, as there is both a known solution, and there does not appear to be widespread understanding of the scope of the issue in the 
field.

The discrete-time assumption is essentially that the processes in question only change and interact when observations occur - only at these moments do the processes suddenly experience random shocks and influence from other processes, with supposedly no change happening in the time period between observations. Although this assumption is not necessarily a problem for causal inference in first-order bivariate scenarios, with anything beyond first order dynamics, or greater than two variables in the system, inferences can become invalid. While it is true that this problem is in some sense simply a specific variant of the general problem that misspecified models usually provide inaccurate estimates, the particular form of misspecification in this case is somewhat unusual and many researchers may not have considered it before.

Violations of the discrete-time assumption can have substantial implications for both classical hypothesis testing approaches, and data driven approaches seeking the temporal structure of processes. The consideration of these two, via numerical example as well as simulation, constitute the main part of this paper. In addition, it becomes apparent when resolving problems with the discrete-time assumption that one cannot observe the system at every moment of change - this makes the known lag assumption, as well as the impact of measurement error, important to address, and discussion on these is found in the latter portion of the paper.

\section{The Discrete-Time Assumption and Misspecified Temporal Structure}

Causal inference regarding cross-lagged parameters has typically occurred in the classical hypothesis testing framework, in which competing models are created based on theory, and their performance compared. A simple example of such could be the earlier question of whether umbrella carrying predicts ice cream sales - a model including an effect of umbrella carrying on sales would be compared to a model where this effect is fixed to zero. In contrast to the theory driven approach, increases in both data availability and computation in recent years have seen the development of data driven approaches. In this case, formal hypotheses are not explicitly specified but instead a model structure is estimated by including a preference for sparsity in the estimation approach - cross-lagged coefficients of zero are preferred. This sparsity preference is achieved by forms of regularization (e.g. the L1 penalty, or lasso, of Tibshirani, 1996), where additional terms are included in the estimation routine that preference smaller and or zero values of cross-lagged coefficients. Without such a sparsity preference, even when the effect of umbrella carrying on sales is truly zero, the estimated coefficient would not be exactly zero due to a limited observation size. Both classical and data driven inference approaches to cross-lagged coefficients are regularly misap- plied in psychological research. Cross-lagged panel models, multivariate change-scores, VAR models, and similar approaches all implicitly assume processes evolve in discrete jumps in time, when in reality researchers tend to think of the underlying processes as continually in flux. While the need for continuous-time systems for inference with stochastic processes has already been discussed somewhat, (see for example Aalen, 1987; Aalen et al., 2016; Deboeck \& Preacher, 2016, Deboeck et al., 2018, Kuiper \& Ryan, 2018, Ryan \& Hamaker, 2022), discrete-time cross-regressive models are still the dominant paradigm for inference in such settings. To explicitly highlight the problem, in this section I provide some examples in the domains of both hypothesis testing, and structural discovery via regularization. The take home message will be that even in relatively sparse causal systems, the temporal regression matrix between observations at different times is very likely to be dense (contain no zeroes), or at least less sparse (contain fewer zeroes). In some cases there can also be similar sparsity (i.e. number of coefficients with value zero) to the true system but in different locations. The fundamental problem is that this discrepancy invalidates attempts to infer structure or direct causal paths via the specification of, or regularization to, zeroes in the temporal regression coefficients. This issue arises due to a difference in how the systems are typically expected to behave, which is continuously (or very frequently) interacting, and how they are modeled, which is interacting only when measured. When the underlying system is continuously changing, this discrepancy can be resolved by directly estimating a continuoustime temporal effects matrix, and specifying or regularizing this to zero - the corresponding discrete-time regression weights and covariances can then be computed based on the continuous time temporal effects, or 'drift', matrix, and the time interval between observations. Important to note, is that this all applies whether or not time intervals vary between observations or subjects.

To facilitate understanding, I will first consider the temporal structure issue in a purely discrete-time context, where change can genuinely be represented as occurring at specific times. When a cross-lagged model represents a hypothesis that a) the processes actually evolve in discrete jumps, and b) the measurement occasions $u$ actually capture each and every one of these discrete jumps, then there is no problem and we can have a perfect representation. To consider such a system, we could imagine 3 people exchanging information by post, and the post is delivered daily. Person $\mathrm{x}$ writes to person $\mathrm{y}$, and person $y$ in turn passes this information on to person $z$. If we know that person $x$ has received new information, we can expect y to become aware of this the following day, while it will take an additional day before $\mathrm{z}$ knows it. In this case, we can precisely represent the causal pathways using a temporal regression matrix (or path diagram) that represents change over a time interval of one day or one postal delivery, as per 
Figure 1. Here, $\mathrm{y}$ is entirely dependent on $\mathrm{x}$ for information, and $\mathrm{z}$ is dependent on $\mathrm{y}$.

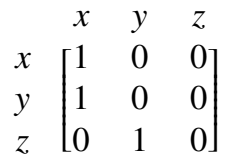

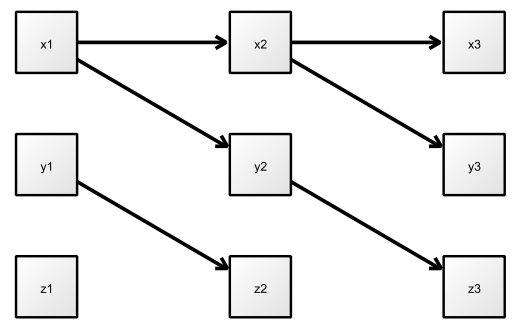

\section{Figure 1}

Representation of daily packets of information flow between persons $x, y$, and $z$, across 3 days.

Using such a representation, our model will perform optimally in terms of prediction, where we can use the knowledge of what information each individual has on a particular day to predict future days. Furthermore, we can also use the structure to understand what will happen if the structure changes - if for instance the link between $\mathrm{y}$ and $\mathrm{z}$ is removed, we know that $\mathrm{z}$ will not receive any information from $\mathrm{x}$.

What happens to our representation if we instead shift to an observation time interval of two days or two deliveries though? As soon as we deviate from the 'true' time step over which information flow occurs, our representation no longer matches the data generating process, or causality, inherent to the system. For an interval of two days, the temporal regression matrix would look as follows, and the path diagram as per Figure 2 When the new time interval is simply an integer $\tau$ multiple of the original, the new temporal regression matrix can be obtained by matrix multiplying the original matrix by itself $\tau-1$ times.

\begin{tabular}{|c|c|c|}
\hline & $x$ & $y$ \\
\hline$x$ & & 0 \\
\hline$y$ & & \\
\hline & & \\
\hline
\end{tabular}

In this case, we have accurately represented how information flows through the system at time intervals of two days, in that once a change in $\mathrm{x}$ occurs, 2 days later we see changes in both y and z. So, we can still use the representation to make accurate predictions on a 2 day interval. However, we clearly have not represented the actual causality in the system! That is, our system does not accurately represent the way in which information flows through the system, and thereby leads to

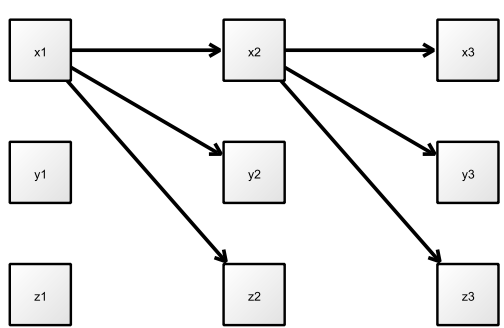

Figure 2

Representation of packets of information flow between persons $x, y$, and $z$, across 6 days, when the time interval is two days.

some incorrect estimates of causal effects under certain (hypothetical) interventions. Some causal effects still turn out accurate, as we see that the effect of a change in person $\mathrm{x}$ on person $\mathrm{z}$ two days later is correct. However, one might also be tempted to infer from this two-day representation that person $y$ is unnecessary for the information flow between $x$ and $\mathrm{z}$, but in fact if we were to intervene on the system and block the route from $y$ to $z$, we would see that no mail reaches $z-$ person $\mathrm{y}$ is required if any information from $\mathrm{x}$ is to reach $\mathrm{z}$.

Considering causality in such a way, where we also think of adding or removing paths between variables, may seem slightly unusual to those familiar with the consideration of causal effects by intervening on variables rather than paths. Under such a variable oriented view, expectations of causal effects can be computed by intervening and setting one of the variables in the system (i.e. $\mathrm{x}, \mathrm{y}$, or $\mathrm{z}$ ) to a specific value, either for a moment or for some length of time (Ryan \& Hamaker, 2022). In the realm of linear models this may seem the only possible approach, however there is no reason to restrict ourselves to linearity here - this is a conceptual consideration and does not have to be included in any model of data for us to understand the concept. In a non-linear system, the system can be expanded to include any of the paths as a variable itself. In the mail example, we could include a fourth (latent) variable to represent the path between person $\mathrm{y}$ and person $\mathrm{z}$, then the proposed intervention of taking $\mathrm{y}$ out of the system would change the coefficient of this added variable from 1.0 to 0.0 .

This discrepancy discussed between the true causal system (or generating model), and the information flow given an arbitrary unit of time, captures a problem inherent to many longitudinal modeling endeavors. Such a discrepancy is precisely why discrete-time cross regression parameters cannot be naively used as the basis of hypothesis tests regarding direct causal paths, or as indicators of some generative structure when achieved via regularization. The causal conclu- 
sions that result can be invalid, even when the true model is some form of VAR and plenty of data is available - unless every possible occasion of change (whether observed or not) is included in the model.

As we can see from the example, problems related to hypothesis tests and structure determination with discrete time models occur even when the true data generating function is a discrete time function, whenever more than one step of change has occurred between observations. Given that most psychological processes tend to be thought of as continuously existing and interacting with each other, rather than interacting only when we decide to measure them, this poses something of a problem, as regular longitudinal structural equation modeling and network modeling approaches cannot represent the data generating processes that researchers typically hypothesize. While this is not inherently problematic when models are used only for predictive purposes - in the postal example, we could still make accurate two-day forward predictions using the two-day temporal regressions it is a problem whenever the underlying causality (i.e., the mechanisms that bring about change), is of interest.

\section{Continuous Time}

Instead of modeling processes as a sequence of discrete jumps, differential equations - the mathematics of continuously changing processes - have been available and widely used in the sciences for hundreds of years. While the fields of psychology have recently been showing more awareness of differential equation approaches, the full ramifications with respect to understanding and testing causal structure still appear little understood. Hansen and Sokol (2014) provide a detailed overview of causal interpretations of continuoustime models, and Maclaren and Nicholson (2021) highlights how stochastic-differential equations can be incorporated into an expanded concept of the typical causal inference frameworks in use. While some of the mathematical computations required for software to solve the equations and fit continuous time models can look cruel and unusual, an intuitive understanding is possible, and can also resolve a range of time-related confusions that can also occur with more familiar regression oriented approaches (Voelkle et al., 2019. For more on such confusions, see).

In discrete-time systems, the matrix of temporal effects represents regression strengths between two points in time, as in the one or two day examples discussed in the postal example. Continuous-time approaches can be intuitively thought of in much the same way, but simply compressing the time interval to a 'very small' value. This allows for usage of a conceptually similar temporal effects matrix, the only difference being that the matrix represents the influence of the current state of the system on the direction of change in the processes (analogous to the approach used in changescore structural equation models, where an autoregression of
1.00 is always applied in addition to any estimated self and cross feedback parameters). This contrasts to the discretetime form where an earlier state of the system is used to predict the new level of the processes. A continuous-time form of the VAR discussed is:

$$
\mathrm{d} y(t)=(A y(t)+b) \mathrm{d} t+G \mathrm{~d} W(t)
$$

This looks similar to the discrete-time form, but instead of telling us the new value of $y$ given one step forward in time, it tells us how $y$ is changing at the moment. Some mathematical complications due to the nature of stochastic differential equations are present: the $\mathrm{d} t$ on the right hand side, which can be thought of as a very small step in time, and the $\mathrm{d} W(t)$, which represents white noise in continuous time. To compute $y$ at some time point given an earlier value, one needs to solve the system. This is numerically involving and described in detail in Voelkle et al. (2012), however approximations using linear extrapolation can be done by hand, and can be quite helpful - sometimes for numerical simulation, but also just to follow the basic logic, which can help give an intuitive sense of what the parameters do in the model.

$$
\left.y_{u}=y_{u-1}+\Delta t\left(A y_{u-1}+b\right)+G z_{u} \quad z \sim N(0, \Delta t)\right)
$$

Essentially then, one computes the deterministic rate of change using $A$ and $b$ and the earlier value of $y$ at observation occasion $u-1$, then multiplies this by the length of time step $\Delta t$ desired. The variance of the white noise element $z$ is then also $\Delta t$, resulting in a system noise (co)variance of $G G^{\top} \Delta t$. Shorter steps are (up to a point) more accurate, but require more computations. Many refinements are possible this only represents the basic idea, which is called the EulerMaruyama method in the context of stochastic differential equations. The linear system shown also allows an 'exact' one-step solution. While this is described in more detail elsewhere (e.g. Driver \& Voelkle, 2018, contains the equations and code for plotting), the basic component involves the matrix exponential, with temporal regression coefficients for particular time intervals given by $e^{A \Delta t}$.

The continuous-time approach becomes important when the underlying system is assumed to be continuously changing and interacting. The exact proportion of psychological variables where this is the case may be an open question, but there are certainly many cases where a continuous assumption is more plausible than a discrete assumption. To illustrate this we can consider a similar system to the postal example, but where interactions occur continuously. In this case we have $x$ (motivation) affecting $y$ (exercise) which in turn affects $\mathrm{z}$ (fitness). The continuous-time temporal matrix is: 


$x$
$y$
$z$$\left[\begin{array}{ccc}-1 & y & z \\ 1 & -1 & 0 \\ 0 & 1 & -1\end{array}\right]$

The negative coefficients on the diagonal tell us that increases in any of the variables exert a downwards pressure on that same variable in the future, which is typical of systems that fluctuate around a certain range rather than exploding out to negative or positive infinity (as can occur with positive diagonals). The positive cross-effects show where change in one variable (determined by the column) leads to positive change in another (determined by the row). Figure 3 illustrates the implied discrete-time coefficients for the effect of a change in $\mathrm{x}$ on all three system variables at later time points, as the time interval changes. This is computed using $e^{A \Delta t}$. Such a plot is also interpretable as an impulse response function (given an impulse of magnitude 1.00 to the causal, i.e. column, variable). The value of $x$ at time zero (i.e. when the change in $\mathrm{x}$ occurs) becomes less predictive of later values of $x$, so the coefficient reduces. Initially, the time zero value of $\mathrm{x}$ is not predictive of either $\mathrm{y}$ or $\mathrm{z}$, but as time goes by the change in $\mathrm{x}$ exerts influence on $\mathrm{y}$, and then via $\mathrm{y}$ on $\mathrm{z}$, so the regression coefficients rise. At some point, once the initial change in $\mathrm{x}$ has worn off to a sufficient degree, the coefficient for $\mathrm{x}$ predicting $\mathrm{y}$ reaches a peak and starts to reduce, and similarly for $\mathrm{z}$. Important to note is that any such peaks do not represent a period of 'peak effect' or the like the cross-effect is constant over time, it is simply that the $a c$ cumulation of any effect becomes most obvious somewhere between the time of the initial change, and the time when the initial change has mostly dissipated.

From Figure 3 we can see that in such a continuous system, the presence of non-zero discrete-time coefficients at any particular point in time does not imply a direct causal link between the variables. If we have a system of motivation to exercise (x), daily exercise levels (y), and fitness (z), a not unreasonable causal hypothesis would look like the structure of Figure 1. where motivation leads to exercise which leads to fitness, or $\mathrm{x}->\mathrm{y}->\mathrm{z}$. Consider then a yearly panel study and a typical discrete-time modeling approach, where $\mathrm{x}$ at the first time point affects $y$ at the second, which in turn affects $\mathrm{z}$ at the third. Mapping the underlying causal structure to the yearly temporal coefficients in such a way implies that changes in motivation take a year to affect exercise, and then more importantly a further year to affect fitness at all. So, our model has encoded in it not only the assumptions that $x$ $->\mathrm{y}-\mathrm{z}$, but also that $\mathrm{x}$ has no effect on $\mathrm{z}$ until two years have passed. If we instead had observations every 6 months, the very same model structure would imply that changes in $\mathrm{x}$ have no effect on $\mathrm{z}$ for only one year instead of two! While Figure 3 is no doubt still inaccurate (because humans are highly complex and it is a made up example using a linear system), it gets closer to what we would expect - an initial

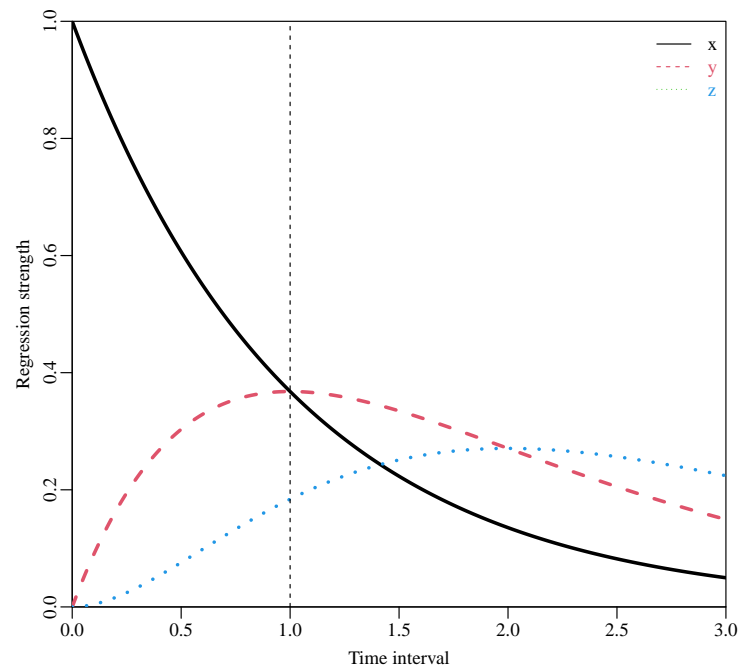

Figure 3

Temporal regression coefficients between $x$ at an earlier point in time and $x, y, z$. Note that the coefficient between $x$ and $z$ is positive, even though there is no causal effect. A time interval of 1 unit is marked - the same temporal matrix structure in a discrete-time approach would still show 0.00 for the influence on $z$ at this point.

increase in motivation (x, black) starts to increase daily exercise levels ( $y$, red), which in turn start to increase fitness levels (z, blue).

So, non-zero coefficients in a discrete-time temporal matrix represent the idea that at some (potentially arbitrary) point in time, one variable is predictive of another. As we see in the exercise example however, this does not readily map to an understanding direct causal paths, as even in the absence of a direct effect, non-zero coefficients are to be expected. In contrast, modeling the continuous-time system directly allows one to turn off (i.e. fix to zero) certain causal paths of the temporal effects $A$ or system noise effects $G$. This point is easy to miss because there are substantial equivalences between discrete and continuous time models when temporal effects are freely estimated, in that with free parameters, equivalent predictions are (in many cases) arrived at. The distinction comes about when restrictions are imposed on the system - zeroes in discrete-time temporal effects at one particular sampling rate do not necessarily translate to zeroes in discrete-time effects given a different sampling rate, or to zeroes in continuous-time temporal effects. For continuously evolving systems, it is often the continuous-time effects that are of interest, as these represent how (predictable) change propagates through the system, while the discrete- 
time effects represent total (predictable) change over a specific length of time.

To highlight the scope of potentially invalid inferences in these directions, I simulated temporal-effects matrices of continuously interacting systems with a range of dimensions and levels of sparsity, observed at time intervals of 1.00. Selffeedback effects (i.e. the diagonals) were randomly generated based on the negative exponent of a standard normal distribution, with cross effects drawn from a normal distribution with mean of 0 and standard deviation of 0.5 . A proportion of cross-effects was then randomly selected according to the level of sparsity, and these were set to 0 . The correct discrete-time temporal regression matrices were computed for each of these systems by taking the matrix exponential of the continuous-time temporal-effects. Systems with explosive tendencies (i.e. where any regression coefficients continue increasing over time due to strong feedback loops) were dropped from the simulation as they were deemed less likely to reflect common psychological scenarios. The computed discrete-time coefficients were then checked for inferential consistency regarding zeroes, as zeroes represent the case of no direct effect between variables. Perfect consistency is found when the pattern of zeroes in the discrete-time matrix exactly matches the pattern of the generative continuoustime system. To reflect the fact that values near zero are often assumed to be, or are regularized to, zero, two arbitrary threshold conditions were used, a stricter 0.01 and more liberal 0.05. Coefficients that were closer to zero than these thresholds were assumed zero. All choices in this simulation are necessarily somewhat arbitrary, but the point is found in the general patterns, not the absolute values. The code provided in supplementary material allows for changes to these decisions if desired. The left column of Figure 4 shows the proportion of reasonably inferred zeroes (i.e. the correct absence of a causal path) when using discrete-time matrices for inference. Note that for some cases the proportion reasonably inferred exceeds the target 'perfect' level, shown as a dashed line, because of the threshold. The right column shows the proportion of unreasonably inferred zeroes (i.e. the incorrect absence of a causal path). For those interested, the same plot but for inference based on the continuous-time system is available in the supplementary material - results are, as should be expected, close to perfect besides some inflation of the reasonably estimated zeroes, due to the use of a non-zero threshold.

From Figure 4 we can also see that the proportion of incorrectly inferred zeroes increases, with higher proportions in dense, highly connected systems. Moreover, as dimensions increase the proportion of zeroes in the discrete-time matrices is too low, except for systems with either mostly zeroes, or no zeroes. This means that when using discrete-time models to represent continuous-time systems a) more parameters are often necessary to estimate the same causal system, and b) cross-effect parameters may be needed between variables that have no direct causal path in the generating system.

Where does this difference in sparsity between continuous and discrete time forms arise from? Is it perhaps simply an artifact of the simulation conditions? The dense(r) temporal networks from the discrete-time form arises because the continuous-time contains only the direct causal paths in the system, while the discrete-time form represents the predictiveness (or 'total' effect) of each variable after some particular length of time. Such predictiveness can come about due to both the direct paths, but also any indirect paths. If we consider again the motivation, exercise, and fitness example, there are direct paths from motivation to exercise, and from exercise to fitness. Once these relations are considered over some length of time, the direct paths still exist, in that earlier motivation predicts later exercise, and earlier exercise predicts later fitness. Additionally, earlier motivation predicts later fitness, because of the indirect path via exercise. Because the discrete-time form represents this expansion over some length of time, it inevitably contains some mixture of these direct and indirect effects, resulting in generally denser temporal relation matrices. It is possible to construct specific cases where this is not so, but they require a very specific balance of effects that are unlikely to be found in reality. Deboeck et al. (2018) and Ryan and Hamaker (2022) describe the relation between direct, indirect, and total effects in continuous and discrete time systems in much greater detail, from the perspective of path tracing in structural equation modelling.

\section{Regularization}

Having highlighted the problems of relying on discretetime matrices as a basis for inference when systems evolve continuously, it is hopefully clear that for many confirmatory modeling and hypothesis testing endeavors, one should instead parameterize the continuous-time temporal effects matrix. From that point, regular tools of hypothesis testing and model comparison can be used as normal. Moving away from confirmatory modeling however, it has become popular within some fields to use regularization to zero (e.g. using the lasso of Tibshirani, 1996) as the basis for inferring causal structure of a system. Some such approaches include the graphical VAR with regularization (Epskamp et al., 2018, Yuen et al., 2018), regularized multivariate latent change scores (Jacobucci \& Grimm, 2018a), and group iterative multiple model estimation (Gates \& Molenaar, 2012). J. J. Park et al. (2020) contrasts some of the different approaches mentioned for the consideration of affect and personality dynamics, while some other example applications include relations between well-being and sleep (Dickens, 2020), and anxiety and borderline personality (Ellison et al., 2020). Historically such approaches have tended to neglect the possibility of measurement error (potentially quite problematic, discussed 


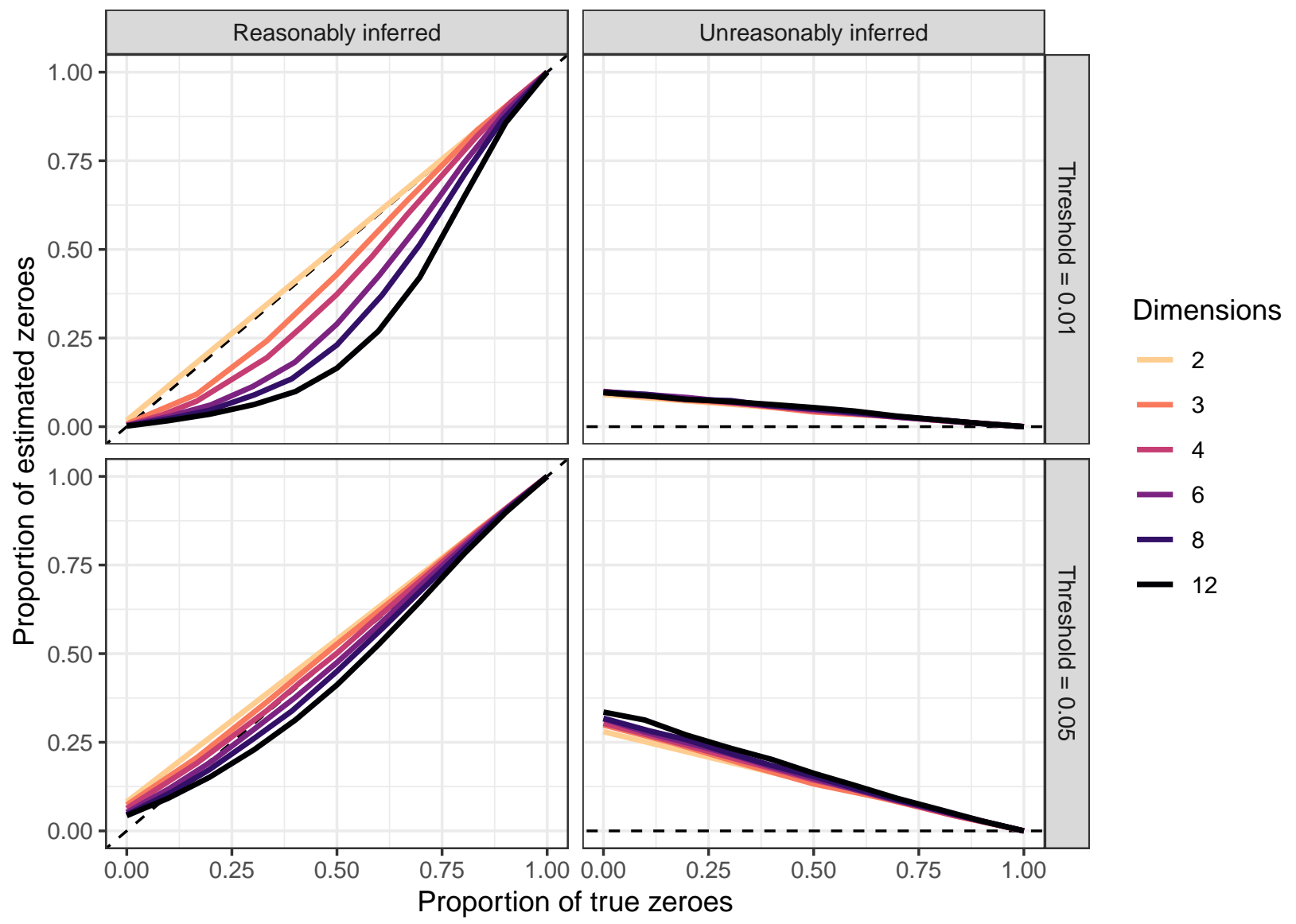

Figure 4

Simulation results, showing how the use of discrete-time models for inference about continuously interacting systems can result in invalid conclusions, across different dimensions, sparsity, and threshold criteria. The dashed line indicates what would be 'perfect' inferences, though note that due to the use of a threshold (below which effects are assumed zero), 'Reasonable' inferences about the existence of zero coefficients are in some cases higher than this. As the dimensionality rises, so too does the propensity for incorrect inference about a given coefficient.

below), though this is changing of late (Bulteel et al., 2018; Epskamp, 2020, Gates et al., 2020; Liang et al., 2021).

The approaches typically used for data-driven discovery have primarily used discrete-time. As is hopefully clear by now, this means that regularization to zero is being applied to the temporal regression coefficients for a specific time interval, and that these do not do not necessarily correspond to zeroes in an underlying continuous-time process. This means that while sparsity may be achieved for the time interval in question, this does not imply sparsity at any other time interval, and the recovered sparse structure does not necessarily represent the underlying generative structure. In addition, given that discrete-time temporal matrices are likely to have more non-zero coefficients than continuous-time, resultant structures are less likely to achieve sparsity goals for comparable levels of regularization. A natural step at this point, assuming the generative structure is of interest, is to regularize the underlying continuous-time temporal-effects matrix instead. The ctsem software (Driver \& Voelkle, 2018) offers the ability to specify Bayesian priors on the system parameters, which, when estimated using the default approach of optimization, are equivalent to typical penalized likelihood approaches used for regularization (for more elaborated discussion on this see Jacobucci \& Grimm, 2018b; T. Park \& Casella, 2008). An alternative approach by Orzek and Voelkle (in press), regCtsem, builds on the original ctsem software based on OpenMx (Neale et al., 2016), now available as ctsemOMX. This makes it more limited in terms of model flexibility, but it includes more emphasis on regularization, specifically if and how standardization of variables or parameters is performed.

The default prior in ctsem (when priors are enabled) is 
a Gaussian distribution, which can be interpreted as L2, or ridge, regression. L2 penalization does not lead to sparse matrices, because coefficients will not, in general, be pushed all the way to zero. In addition to this, large coefficients are heavily penalized. A common fix for these issues is to replace the Gaussian prior with a Laplace distribution (interpretable as L1 penalization or lasso regression), ensuring that small coefficients will be pushed to zero, and large coefficients are not affected as heavily. As discussed in Orzek et al. (2022), a smoothed form of L1 penalization is implemented in ctsem removing typical optimization difficulties with standard L1 penalization.

A comparison between L1 regularization on a discrete versus continuous time representation is shown in Figure 5. The underlying system of four processes is a causal chain of the sort already discussed, where the first process causes the second and so on. Random noise affects the processes independently with the same magnitude. These processes do not evolve in discrete-jumps, but change and interact continuously. The continuous $(A)$ and corresponding discrete $\left(e^{A}\right)$ time temporal relations matrices looks like:

$$
A=\begin{array}{cccc}
-1 & 0 & 0 & 0 \\
0.5 & -1 & 0 & 0 \\
0 & .5 & -1 & 0 \\
0 & 0 & .5 & -1
\end{array} e^{A}=\begin{array}{ccccc}
0.37 & 0 & 0 & 0 \\
0.18 & 0.37 & 0 & 0 \\
0.05 & .18 & 0.37 & 0 \\
0.01 & 0.05 & .18 & 0.37
\end{array}
$$

Data was generated for 10 subjects with 100 observation occasions each, sampled at time intervals of 1.0. Figure 5 shows how estimated temporal coefficients of the system change as the penalty (i.e. width of the prior) changes, for both a discrete and continuous time estimation approach. Only the cross-effects that are zero in the generating model are shown (for simplicity). The penalty at which the best out of sample (subject-wise) predictive performance of the model was achieved is shown by the black dotted line. Note that because the scale of the discrete and continuous coefficients are not necessarily the same, the ideal penalty for out of sample prediction for each model will also not necessarily be the same. From the figure, we see the expected shrinkage of coefficients towards zero. At the point of best out of sample performance, the discrete-time model has more nonzero coefficients. This result is unsurprising, and should be expected based on the true discrete-time matrix shown above - nevertheless this hopefully gives a more tangible sense of the problem to researchers in the field considering these approaches.

\section{'True' Time Lag and the Importance of Measurement Er- ror}

A common question from researchers designing a study is at what speed the system is changing, what is the 'true' time lag of the system. As highlighted by Shojaie and Fox (2022),

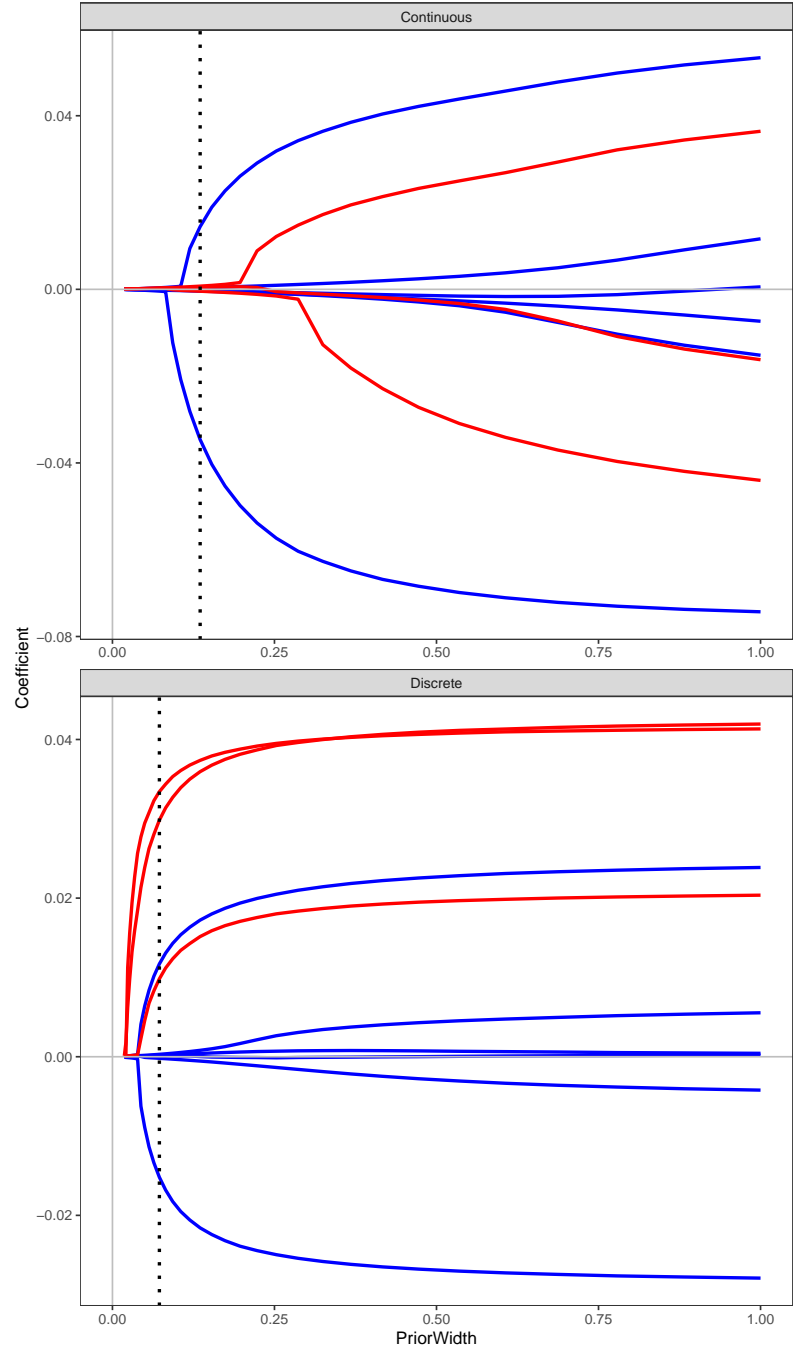

Figure 5

Estimated temporal coefficients in a 4 dimensional system, using L1 (lasso / Laplace prior) penalization with different penalties (prior widths). Both continuous and discrete time models were estimated. All coefficients shown were zero in the continuous-time generating model, those that were nonzero in the discrete-time representation with a time step of 1.0 are colored red. Best out of sample performance for each model is indicated by the vertical black dotted line. Note the (expected) presence of more non-zero coefficients in the discrete-time estimation.

this is a critical consideration if using a standard discretetime VAR type model to represent a discrete-time system the true time lag of the system needs to match the time interval of the observation procedure. This has led to works that focus on specifying the most appropriate time interval (e.g., Dormann \& Griffin, 2015, Hopwood et al., 2022), as well 
as those that aim to specify the correct lag structure (e.g., Jacobs \& Otter, 2008, Jacobson et al., 2019). This need to observe at the same rate the system is changing may seem quite problematic for a system that continuously fluctuates, as we can generally not observe phenomena continuously, but take snapshots of the system state at specific moments in time. Fortunately, this assumption that the true time lag matches the observation rate is easily relaxed when the underlying system is continuous-time - so long as measurement error is accounted for.

The importance of accounting for measurement error in discrete-time VAR type models is well documented (Schuurman \& Hamaker, 2019, Schuurman et al., 2015, Staudenmayer \& Buonaccorsi, 2005), as ignoring measurement error can lead to downwards biased auto-regression coefficients and potentially spurious cross-regression relationships. This all holds true for the continuous-time case also. A related issue that does not seem widely understood is that when measurement error is ignored, the apparent time scale of the underlying processes (inferred from estimates) can depend strongly on the frequency at which observations are obtained. This dependence can make the selection of sampling frequency (e.g. weekly vs monthly) seem a crucial design decision. Fortunately, once measurement error is accounted for the choice of sampling rate is less crucial, and becomes instead a matter of maximizing information gain per observation, rather than the observation design being inherently wrong and inevitably leading to the wrong conclusions. For more detail on the information gain aspect and various trade-offs regarding target parameters and the relation to sampling rate, see Adolf et al. (2021) and Timmons and Preacher (2015).

A common approach to handling measurement error is to include a Gaussian measurement model of the processes. In most basic form this might look like:

$$
\mathbf{x}_{u}=\mathbf{y}_{u}+\boldsymbol{\epsilon}_{u} \quad \text { where } \boldsymbol{\epsilon}(t) \sim \mathrm{N}(\mathbf{0}, \boldsymbol{\Theta})
$$

Where $\mathbf{x}_{u}$ now represents the observations at measurement $u$ and $\mathbf{y}_{u}$ the latent processes, measured with error $\boldsymbol{\epsilon}_{u}$. The errors are random with variance(s) given by $\boldsymbol{\Theta}$. This can be handled using structural equation or state-space modeling approaches, as used in the already mentioned software options. This model is often extended to a linear factor model with a factor loading matrix and intercepts (e.g., as in Driver \& Voelkle, 2018).

To demonstrate the problematic phenomena discussed regarding measurement error and sampling rate, I simulated data for 500 subjects with 30 measurement occasions each, based on a hypothetical model where language abilities facilitate mathematics ability development, but not the reverse, and variables are observed with Gaussian measurement errors. Subjects were observed either every 0.2 or 0.6 years. These sampling rates were crossed with model specification.
For one model, measurement error variance was assumed non-existent, while for the other it was freely estimated. Figure 6 shows the results of this, with data fit using the ctsem software (Driver \& Voelkle, 2018). These plots show the core points mentioned, with the top row (wrongly assuming no measurement error) showing: spurious (not genuinely causal) cross effects from maths ability to language (green and purple curves in top right); precise but incorrect (too fast) estimates of temporal relations generally; and differential results that are predictable based on the sampling interval while the orange / pink and green / purple curves represent the same underlying phenomenon, the curves from the more frequent observation regime show faster effects. The lower half of the figure, where measurement error is appropriately included in the estimation model, shows approximately correct estimates (the true values are included in the confidence regions shown), and the sampling frequency does not impact the time scale of estimated processes.

Two caveats are important to emphasize here. While accounting for measurement error can avoid the dependency of estimates on the sampling rate, as already mentioned the information gained from each measurement for various parameter estimates will still depend on a complex function depending in part on the amount of true change and measurement error in the observation. One will struggle to estimate slow yearly dynamics with microsecond based estimates over a minute, just as one will not capture microsecond dynamics with yearly sampling. The second caveat is that although we do not need to know the 'true lag' of the system, 'lag' and 'order' are terms with important differences that are sometimes confused. The lag of a system refers to how many steps in time before a change in one variable predicts a change in another, while the order of a system refers to the number of interrelated processes needed to represent the dynamics of one observed variable. In many discrete-time estimation approaches lag and order can be treated interchangeably, but this is not the case when it comes to continuous-time. Estimating a model where the order of the system is too low is a problem - the model is not sufficiently complex to capture the dynamics and may lead to invalid inferences and poor predictions. Substantial work has occurred in this domain, I will point to only a few examples, and highlight one approach to model checking that uses discrete-time model formulations.

\section{True Model Order and Structure}

While the majority of psychology oriented research with VAR type models relies on first order linear approaches, more complex models have been explored from time to time, particularly oscillatory models (for some examples see Boker et al., 2016, Chow et al., 2005, Hu et al., 2014; Oud et al., 2018). Non, or semi, parametric approaches are often a useful tool to check and or explore system struc- 


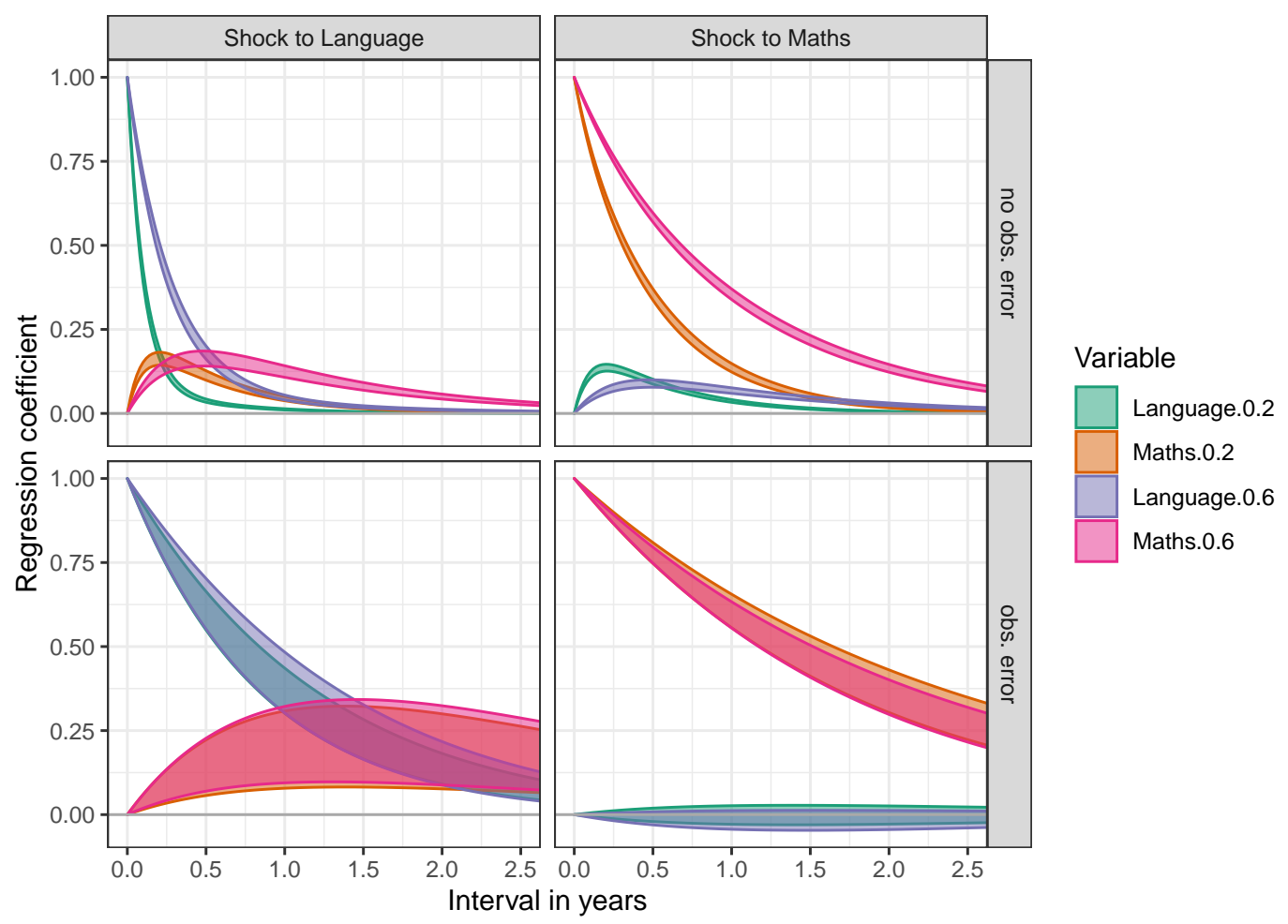

Figure 6

Discrete-time auto and cross regression 95\% confidence regions estimated based on either a correctly specified continuoustime model (bottom row) or the same model but without accounting for measurement error (top row). The variable types are suffixed with the sampling time intervals of either 0.2 or 0.6, but as there is no difference in the underlying process both the orange / pink, and green / purple, regions should overlap, as seen in the correctly specified lower row. The top row where measurement error is not accounted for shows spurious cross-effects, precise but inaccurate estimates, and changes in estimated time-scale (i.e. slower / faster) depending on sampling rate. These problems do not occur once measurement error is accounted for, as in the lower row.

ture, as they are less dependent on specific assumptions, and Chow (2019) discusses the use of such approaches for model development. Parametric approaches are also possible, Belcher et al. (1994) provides an alternative parameterization of continuous-time models that allows for simplified model order assessment, and Ha and Welsh (2015) demonstrates model-order selection using regularization approaches. Whichever route is chosen, there is substantial complexity to accurate model development. To convey some basic understanding of the issue, I will demonstrate one relatively straightforward approach to perform at least limited order checking. This approach is just to compare results from a continuous-time model fit with those of a discretetime model (Sims, 2010). This is because for the same model order, discrete-time models can generate more flexible predictions, as they are not constrained to interpolate between observations in any specific way. That is, the very feature that makes discrete-time models at times problematic for inferential purposes, makes them useful descriptively. While not comprehensive, in at least some software (e.g. ctsem from Driver \& Voelkle, 2021) the change between discrete and continuous time requires only a single argument change. Note that this approach may lead to interpretation difficulty if there is substantial variation in time intervals between observations, as the discrete-time approach does not directly accommodate this.

To briefly demonstrate both the impact of a severely misspecified model order (i.e. when substantially different conclusions result) I simulated data from both a first order continuous-time system, and a second order continuous-time system in the form of a damped linear oscillator (Boker et al., 2020, Voelkle \& Oud, 2013). These data were then fit using both first and second order models, in both continuous and discrete time formulations. $\mathrm{R}$ code for this is found in the supplementary material, and the basic results are shown in Figure 7. In the diagonals of the figure we can see the estimated auto regression coefficients when the model order corresponds to the true generating order. The differences be- 


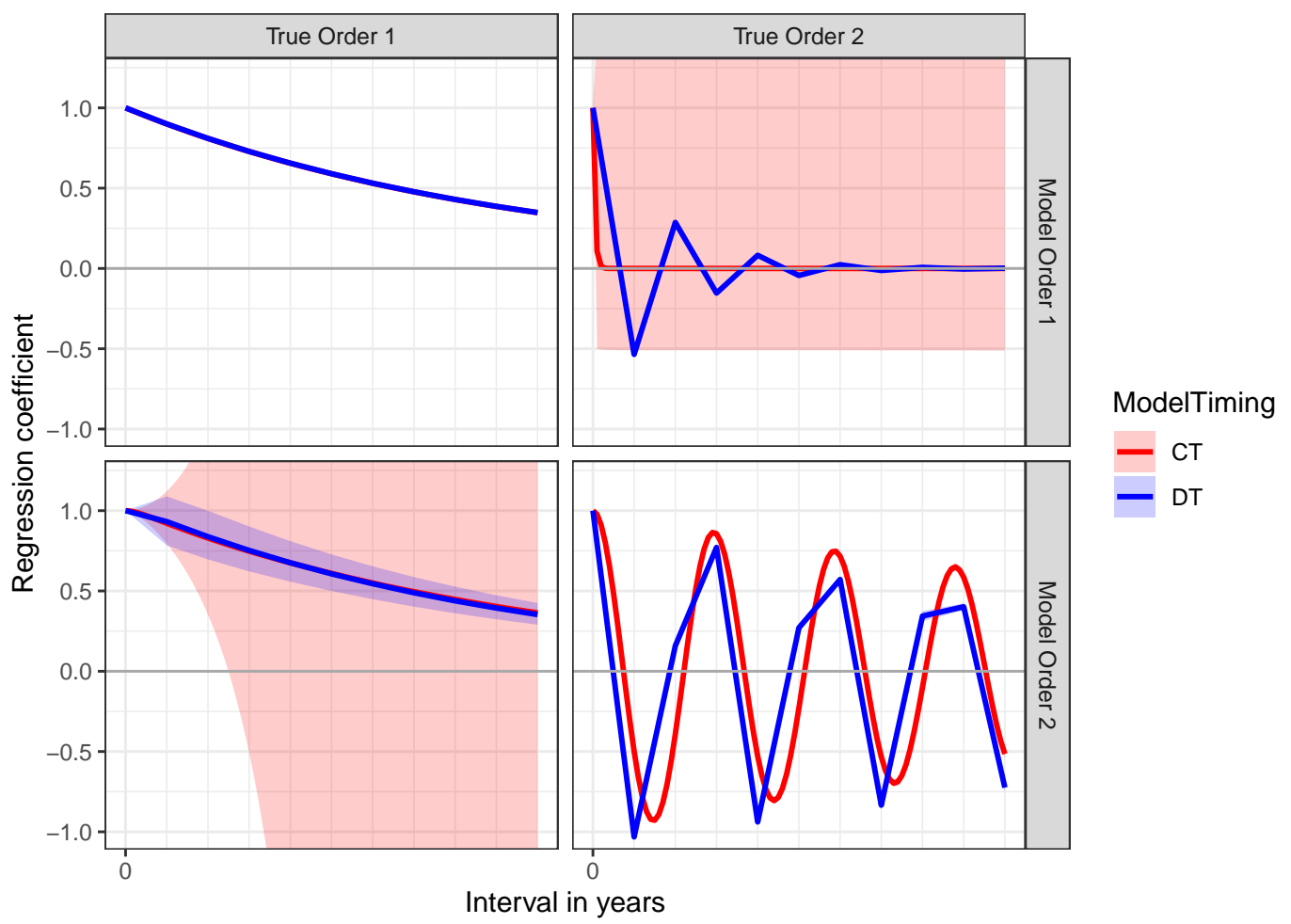

Figure 7

Discrete-time auto regression $95 \%$ confidence regions and point estimates, from either a 1st (top row) or a 2 nd (bottom row) order model. Models are fit to simulated data from either a 1st (left column) or 2nd (right column) order continuous-time process.

tween continuous and discrete time models in the lower right is due to the use of linear interpolation - the grid lines denote where observations actually occurred. In the top right, we see that when a first order continuous-time model is used as a model for a true second order process, and this process results in consistent oscillations, the point estimates for regression coefficients are quite terrible (comparing to the results from the correct model for an order two process in the lower right). The point estimates for the discrete-time order one model differ quite substantially, although they are still incorrect. A stark difference between the continuous and discrete time models when the order is too low, is the uncertainty - we see for the continuous-time form that the 95\% confidence intervals give an indication that something may be wrong, while the discrete-time coefficients have very little uncertainty about them. While the uncertain parameter estimates from the continuous-time form might provoke a researcher to investigate alternative models, here the discrepancy between the continuous and discrete time conclusions should definitely motivate deeper investigation. Regarding the lower left plot, in which overly complex models are used: point estimates are reasonable (in comparison to the approximately true values in the top left plot), but uncertainty is much higher, particularly for the continuous-time form.

Important to note that such an approach is demonstrated here to convey the core idea. While continuous-time model formulations may link more clearly with our theoretical ideas about how processes change and enable clearer hypothesis tests, more flexible model formulations can provide useful checks of any such theoretically-based model. Switching to the discrete-time model instead of continuous is not guaranteed to even catch such first vs second order violations as shown here, as it will again depend on the observation frequency - more sophisticated approaches as discussed above are advised.

\section{Non-directed Relations}

Throughout the paper so far I have focused on directed relationships, but in many circumstances researchers may instead be interested in covariance, correlation, or partialcorrelation parameters. The same general pattern of results already described holds, wherein zeroes in an underlying continuous system do not necessarily translate to zeroes in a discrete-time estimate. I will not treat this in depth, but for one example consider a two dimensional system with di- 
rect positive feedback effects between processes, and uncorrelated system noise:

$$
A=\left(\begin{array}{cc}
-1 & 0.4 \\
0.4 & -1
\end{array}\right) \quad G=\left(\begin{array}{ll}
1 & 0 \\
0 & 1
\end{array}\right)
$$

The discrete-time VAR for a time interval of 1.0 in this case has a system-noise correlation of 0.27 , even though the generating system noise is uncorrelated. Such issues are part of the motivation behind 'structural' VAR approaches that include both directed and non-directed relations for the system noise in discrete-time - see Chow et al. (in press, 2022) for the link between continuous-time and structural VAR approaches.

\section{Discussion}

In this work I have focused on the problems that using discrete-time representations of continuous-time systems can cause, when inference regarding the underlying processes is of interest. When parameters are freely estimated, discretetime estimates can provide a perfectly valid representation of expected change in a system over a specific length of time, and can be used to estimate the effects of certain hypothetical interventions accurately. In the motivation / exercise / fitness example for instance, a freely estimated discrete-time temporal matrix could provide an accurate estimate of the influence of changing motivation on fitness two years later. They in general cannot be relied upon for typical usages wherein non-zero or zero cross-effect parameters are used to infer the presence or absence of a direct causal relation. Turning again to the motivation example, even though the relation between motivation and fitness was completely mediated by exercise, the cross-regression from motivation to fitness after one year was not zero. These problems also affect efforts to regularize temporal relation parameters - for a given level of sparsity in a continuous-time system (i.e. the number of variables with no direct relation), discrete-time systems tend to be less sparse, and the zeroes that are found may not represent zeroes in the underlying system relationships.

Parameterizing the system as a stochastic-differential equation allows for a truly continuous-time representation, and offers a solution to the problems associated with relying on discrete-time representations for inference about continuous-time systems. Confronted with such problems however, an alternate approach might be to decide the task of accurately specifying and estimating such a system is simply too difficult - there are certainly instances where this may be the most effective option, and if this outcome were all this paper achieved it would be no bad thing. The hope contained in this paper is not to offer any complete package of causal inference from observational data. Rather, the aim is to point to the substantial confusion related to discrete-time representations, and with the remaining sections on regularization, observational time lag, and model order, to point out a general path forwards for those taking the step from discrete to continuous-time models, for those aiming for more realistic representations of the underlying systems.

In the section on true time lag and the importance of measurement error, I discussed the known lag assumption described by Shojaie and Fox (2022), in which the system needs to be observed at the rate that it changes, and that it may be difficult to reconcile with continuous-time systems. Then I demonstrated that once measurement error is accounted for, the estimated dynamics of a continuous-time system are not strongly influenced by sampling rate - so long as the sampling rate is slow enough to distinguish some change from observational error, and fast enough that temporal relations are still detectable.

Time lag and model order are often the same in discretetime systems, but this is not the case for continuous-time. While a full treatment of potential forms of model specification and how to determine such is a substantial task and well beyond the scope here, both alternative parametric and non or semi-parametric approaches can serve as valuable checks and or model development guides. The use of a same order discrete-time model as a contrast to the more parametric (so far as time is concerned) continuous-time form is one of the simpler, but limited, approaches to the problem. Chow (2019) offers many good suggestions here.

Beyond the issues discussed so far, unmeasured confounders are recognized as a major threat to any attempt at system identification and or causal inference. There are a range of approaches to handle the problems posed by unmeasured time invariant, or at least 'slow' changing, variables, (Balestra \& Nerlove, 1966) and these have recently received more attention in the psychological domain (Hamaker et al., 2015, Lüdtke \& Robitzsch, 2022). The ctsem software in use throughout this work allows for both measured and unmeasured time invariant effects across all system and measurement parameters, originally only via Bayesian estimation (Driver \& Voelkle, 2018) but for some time available also via maximum likelihood. Unmeasured time-varying variables are much more problematic, and substantial consideration should be given to such possibilities whenever causal relations between variables in longitudinal studies is of interest. For a review of approaches to measured time-varying confounding, see Clare et al. (2019), while Lubin et al. (2018), Luiz and Cabral (2010), and Streeter et al. (2017) consider possibilities when the unmeasured case is to be taken seriously.

A variety of software is available for fitting data to continuous-time systems with stochastic elements, as described here. Of those targeting social science fields, the $\mathrm{R}$ software packages ctsem (Driver et al., 2017) and dynr (Ou et al., 2019) are purpose built for addressing such systems as described here as well as non-linear variants, and OpenMx 
(Neale et al., 2016) offers functionality for linear dynamic models within its broader scope. Between these three, ctsem includes more direct support for multilevel and or Bayesian approaches, dynr includes regime-switching support, and OpenMx is based on an extended structural equation modelling framework allowing a range of model choices.

Beyond the more conceptual and theoretical concerns discussed in this work, many practical questions arise when a switch from discrete to continuous time representations is considered. A full treatment is well beyond scope, but two very common concerns include computational demands and data requirements. Continuous-time models are computationally more demanding, particularly when for example calculations cannot be re-used across subjects due to the inclusion of individual differences or variable time intervals (Driver, 2021). When such calculations can be re-used, time points are very few and numbers of subjects very high, structural equation model based approaches as offered by ctsemOMX (the original form of ctsem presented in Driver et al., 2017) tend to be fastest - though such an approach quickly reaches its limit as variability is introduced or time points increase. State space approaches (as offered by ctsem, dynr, and OpenMx) chunk computations into more, smaller blocks, and are thus more tractable as complexity or time points rise. Important to consider is that common discrete-time usages are often optimized for simpler cases such as assuming zero measurement error, which can make computational demand differences seem even greater than they are.

The question of 'how much data do I need' is an extremely common one, and there is rarely a good answer - at least though the answer is not very different to discrete-time approaches. If similar model structures are used to answer similar questions, a continuous-time approach may even need somewhat less data as the system is a little more constrained, and time interval variability may help to distinguish between aspects such as stable individual differences, genuine process change, and measurement error. In general, simulations are useful both as a means to familiarize with the different representation, and to determine power under various circumstances.

\section{Conclusion}

Advances in data collection technologies have led to an explosion in longitudinal studies and interest in modeling approaches. Cross-lagged effect parameters have provided both substantial interest and substantial controversy for many years. In this work I have described issues related to problematic interpretation of discrete-time cross effects as directly causal, when the underlying system is in fact continually changing. This issue can be resolved and leveraged by applying more appropriate models such as offered by the ctsem (Driver \& Voelkle, 2018), dynr (Ou et al., 2019) and OpenMx (Neale et al., 2016) software packages, amongst others. The critique and solutions provided here are but one small piece of the puzzle when it comes to accurate representations of psychological systems, but one may hope that the vast increases in data, coupled with improvements in computational performance and many such incremental works as this, do slowly lead to improved representations - the more accurate are our representations of psychological systems, the better we can generalize to new circumstances, times, and interventions.

\section{References}

Aalen, O. O. (1987). Dynamic modelling and causality. Scandinavian Actuarial Journal, 1987(3-4), 177190.

Aalen, O. O., Røysland, K., Gran, J. M., Kouyos, R., \& Lange, T. (2016). Can we believe the DAGs? A comment on the relationship between causal DAGs and mechanisms. Statistical methods in medical research, 25(5), 2294-2314.

Adolf, J. K., Loossens, T., Tuerlinckx, F., \& Ceulemans, E. (2021). Optimal sampling rates for reliable continuous-time first-order autoregressive and vector autoregressive modeling. Psychological Methods, 26, 701-718. https : // doi . org / 10 . 1037/ met0000398

Asparouhov, T., Hamaker, E. L., \& Muthén, B. (2018). Dynamic Structural Equation Models. Structural Equation Modeling: A Multidisciplinary Journal, 25(3), 359-388. https://doi.org/10.1080/10705511. 2017.1406803 _eprint: https://doi.org/10.1080/10705511.2017.1406803.

Balestra, P., \& Nerlove, M. (1966). Pooling cross section and time series data in the estimation of a dynamic model: The demand for natural gas. Econometrica, 34(3), 585-612. https://doi.org/10.2307/1909771

Belcher, J., Hampton, J. S., \& Wilson, G. T. (1994). Parameterization of Continuous Time Autoregressive Models for Irregularly Sampled Time Series Data. Journal of the Royal Statistical Society. Series B (Methodological), 56(1), 141-155 00049.

Boker, S. M., Moulder, R. G., \& Sjobeck, G. R. (2020). Constrained Fourth Order Latent Differential Equation Reduces Parameter Estimation Bias for Damped Linear Oscillator Models. Structural Equation Modeling: A Multidisciplinary Journal, 27(2), 202-218. https://doi.org/10.1080/10705511. 2019.1641816 _eprint: https://doi.org/10.1080/10705511.2019.1641816.

Boker, S. M., Staples, A. D., \& Hu, Y. (2016). Dynamics of change and change in dynamics. Journal of PersonOriented Research, 2(1-2), 34-55. https://doi.org/ 10.17505/jpor.2016.05 
Bringmann, L. F., Hamaker, E. L., Vigo, D. E., Aubert, A., Borsboom, D., \& Tuerlinckx, F. (2017). Changing dynamics: Time-varying autoregressive models using generalized additive modeling. Psychological methods, 22(3), 409.

Bulteel, K., Tuerlinckx, F., Brose, A., \& Ceulemans, E. (2018). Improved Insight into and Prediction of Network Dynamics by Combining VAR and Dimension Reduction. Multivariate Behavioral Research, 53(6), 853-875. https://doi.org/10.1080/ 00273171.2018 .1516540 _eprint: https://doi.org/10.1080/00273171.2018.151654Driver,

Campbell, D. T. (1963). From description to experimentation: Interpreting trends as quasi-experiments. Problems in measuring change, 212242.

Chow, S.-M., Losardo, D., Park, J., \& Molenaar, P. C. M. (in press, 2022). Continuous-time dynamic models: Connections to structural equation models and other discrete-time models. In R. H. Hoyle (Ed.), Structural equation modeling: Concepts, issues, and applications. Guilford.

Chow, S.-M. (2019). Practical Tools and Guidelines for Exploring and Fitting Linear and Nonlinear Dynamical Systems Models. Multivariate Behavioral Research, 54(5), 690-718. https : //doi.org/10.1080/00273171.2019.1566050 _eprint: https://doi.org/10.1080/00273171.2019.156605Driver,

Chow, S.-M., Ram, N., Boker, S. M., Fujita, F., \& Clore, G. (2005). Emotion as a thermostat: Representing emotion regulation using a damped oscillator model. Emotion, 5(2), 208-225. https ://doi .org/ 10.1037/1528-3542.5.2.208

Clare, P. J., Dobbins, T. A., \& Mattick, R. P. (2019). Causal models adjusting for time-varying confounding-a systematic review of the literature. International Journal of Epidemiology, 48(1), 254-265. https:// doi.org/10.1093/ije/dyy218

Deboeck, P. R., \& Preacher, K. J. (2016). No need to be discrete: A method for continuous time mediation analysis. Structural Equation Modeling: A Multidisciplinary Journal, 23(1), 61-75.

Deboeck, P. R., Preacher, K. J., \& Cole, D. A. (2018). Mediation Modeling: Differing Perspectives on Time Alter Mediation Inferences. In K. van Montfort, J. H. L. Oud, \& M. C. Voelkle (Eds.), Continuous Time Modeling in the Behavioral and Related Sciences (pp. 179-203). Springer International Publishing. https://doi.org/10.1007/978-3-319-772196_8

Degtiar, I., \& Rose, S. (2021, February 23). A Review of Generalizability and Transportability. https:// doi . org/10.48550/arXiv.2102.11904
Dekkers, O. M., von Elm, E., Algra, A., Romijn, J. A., \& Vandenbroucke, J. P. (2010). How to assess the external validity of therapeutic trials: A conceptual approach. International Journal of Epidemiology, 39(1), 89-94. https://doi.org/10.1093/ije/dyp174

Dickens, C. (2020). Modeling Sleep and Psychological WellBeing Dynamics in Daily Life using Group Iterative Multiple Model Estimation [Doctoral dissertation, The Pennsylvania State University].

Dormann, C., \& Griffin, M. A. (2015). Optimal time lags in panel studies. Psychological methods, 20(4), 489. C. C. (2021). Computational efficiency in continuous (and Discrete!) time models - Comment on Hecht and Zitzmann. Structural Equation Modeling: A Multidisciplinary Journal, O(0), 1-3. https : //doi.org/10.1080/10705511.2021.1877547 _eprint: https://doi.org/10.1080/10705511.2021.1877547.

Driver, C. C., Oud, J. H. L., \& Voelkle, M. C. (2017). Continuous time structural equation modeling with r package ctsem. Journal of Statistical Software, Articles, 77(5), 1-35. https://doi.org/10.18637/jss.v077.i05

Driver, C. C., \& Voelkle, M. C. (2017). Introduction to hierarchical continuous time dynamic modelling with ctsem. $R$ package Vignette. Available online at: https://cran. r-project. org/web/packages/ctsem/index. html.

C. C., \& Voelkle, M. C. (2018). Hierarchical Bayesian continuous time dynamic modeling. Psychological Methods, 23(4), 774.

Driver, C. C., \& Voelkle, M. C. (2021, January 1). Hierarchical continuous time modeling. In J. F. Rauthmann (Ed.), The Handbook of Personality Dynamics and Processes (pp. 887-908). Academic Press. https:// doi.org/10.1016/B978-0-12-813995-0.00034-0

Ellison, W. D., Levy, K. N., Newman, M. G., Pincus, A. L., Wilson, S. J., \& Molenaar, P. (2020). Dynamics among borderline personality and anxiety features in psychotherapy outpatients: An exploration of nomothetic and idiographic patterns. Personality Disorders: Theory, Research, and Treatment, 11(2), 131.

Epskamp, S. (2020). Psychometric network models from time-series and panel data. Psychometrika, 85(1), 206-231. https ://doi.org/10.1007/s11336-02009697-3

Epskamp, S., Waldorp, L. J., Mõttus, R., \& Borsboom, D. (2018). The Gaussian Graphical Model in Cross-Sectional and Time-Series Data. Multivariate Behavioral Research, 53(4), 453-480. https://doi.org/10.1080/00273171.2018.1454823 _eprint: https://doi.org/10.1080/00273171.2018.1454823.

Gates, K. M., Fisher, Z. F., \& Bollen, K. A. (2020). Latent variable GIMME using model implied instrumental 
variables (MIIVs). Psychological Methods, 25(2), 227-242. https://doi.org/10.1037/met0000229

Gates, K. M., \& Molenaar, P. C. M. (2012). Group search algorithm recovers effective connectivity maps for individuals in homogeneous and heterogeneous samples. NeuroImage, 63(1), 310-319. https://doi.org/ 10.1016/j.neuroimage.2012.06.026

Granger, C. W. J. (1969). Investigating Causal Relations by Econometric Models and Cross-spectral Methods. Econometrica, 37(3), 424-438. https://doi.org/10. 2307/1912791

Granger, C. W. J. (1980). Testing for causality: A personal viewpoint. Journal of Economic Dynamics and Control, 2, 329-352. https://doi.org/10.1016/ 0165-1889(80)90069-X

Ha, H., \& Welsh, J. S. (2015). Model order selection for continuous time instrumental variable methods using regularization. 2015 54th IEEE Conference on Decision and Control (CDC), 771-776. https://doi. org/10.1109/CDC.2015.7402323

Hamaker, E. L., Kuiper, R. M., \& Grasman, R. P. P. P. (2015). A critique of the cross-lagged panel model. Psychological Methods, 20, 102-116. https://doi.org/10. 1037/a0038889

Hansen, N., \& Sokol, A. (2014). Causal interpretation of stochastic differential equations. Electronic Journal of Probability, 19, 1-24. https://doi.org/10.1214/ EJP.v19-2891

Hlaváčková-Schindler, K., Paluš, M., Vejmelka, M., \& Bhattacharya, J. (2007). Causality detection based on information-theoretic approaches in time series analysis. Physics Reports, 441(1), 1-46. https://doi. org/10.1016/j.physrep.2006.12.004

Hopwood, C. J., Bleidorn, W., \& Wright, A. G. (2022). Connecting theory to methods in longitudinal research. Perspectives on Psychological Science, 17(3), 884894.

Hu, Y., Boker, S., Neale, M., \& Klump, K. L. (2014). Coupled latent differential equation with moderators: Simulation and application. Psychological Methods, 19(1), 56-71. https :// doi . org / 10 . 1037/ a0032476 00001.

Jacobs, J. P. A. M., \& Otter, P. W. (2008). Determining the Number of Factors and Lag Order in Dynamic Factor Models: A Minimum Entropy Approach. Econometric Reviews, 27(4-6), 385-397. https : //doi.org/10.1080/07474930801960196 _eprint: https://doi.org/10.1080/07474930801960196.

Jacobson, N. C., Chow, S.-M., \& Newman, M. G. (2019). The Differential Time-Varying Effect Model (DTVEM): A tool for diagnosing and modeling time lags in intensive longitudinal data. $\mathrm{Be}$ - havior Research Methods, 51(1), 295-315. https : //doi.org/10.3758/s13428-018-1101-0

Jacobucci, R., \& Grimm, K. J. (2018a). Regularized Estimation of Multivariate Latent Change Score Models. In Longitudinal Multivariate Psychology. Routledge.

Jacobucci, R., \& Grimm, K. J. (2018b). Comparison of Frequentist and Bayesian Regularization in Structural Equation Modeling. Structural Equation Modeling: A Multidisciplinary Journal, 25(4), 639-649. https://doi.org/10.1080/10705511.2017.1410822 _eprint: https://doi.org/10.1080/10705511.2017.1410822.

Kuiper, R. M., \& Ryan, O. (2018). Drawing conclusions from cross-lagged relationships: Re-considering the role of the time-interval. Structural Equation Modeling: A Multidisciplinary Journal, 25(5), 809-823.

Lewis, D. (1973). Causation. Journal of Philosophy, 70(17), 556-567. https://doi.org/10.2307/2025310

Liang, M., Koslovsky, M. D., Hébert, E. T., Kendzor, D. E., Businelle, M. S., \& Vannucci, M. (2021). Bayesian continuous-time hidden Markov models with covariate selection for intensive longitudinal data with measurement error. Psychological Meth$o d s$, No Pagination Specified-No Pagination Specified. https://doi.org/10.1037/met0000433

Lubin, J. H., Hauptmann, M., \& Blair, A. (2018). Indirect adjustment of relative risks of an exposure with multiple categories for an unmeasured confounder. Annals of Epidemiology, 28(11), 801-807. https://doi. org/10.1016/j.annepidem.2018.09.003

Lüdtke, O., \& Robitzsch, A. (2022). A Comparison of Different Approaches for Estimating Cross-Lagged Effects from a Causal Inference Perspective. Structural Equation Modeling: A Multidisciplinary Journal, O(0), 1-20. https :// doi . org/10 . 1080/ 10705511.2022.2065278 _eprint: https://doi.org/10.1080/10705511.2022.2065278.

Luiz, R. R., \& Cabral, M. D. B. (2010). Sensitivity analysis for an unmeasured confounder: A review of two independent methods. Revista Brasileira de Epidemiologia, 13, 188-198. https:// doi .org/10.1590/ S1415-790X2010000200002

Maclaren, O. J., \& Nicholson, R. (2021). Models, identifiability, and estimability in causal inference. 38th International Conference on Machine Learning. Workshop on the Neglected Assumptions in Causal Inference. ICML.

McArdle, J. J. (2001). A latent difference score approach to longitudinal dynamic structural analyses. Structural equation modeling: Present and future, 342-380.

Neale, M. C., Hunter, M. D., Pritikin, J. N., Zahery, M., Brick, T. R., Kirkpatrick, R. M., Estabrook, R., Bates, T. C., Maes, H. H., \& Boker, S. M. (2016). 
OpenMx 2.0: Extended structural equation and statistical modeling. Psychometrika, 81(2), 535-549. https://doi.org/10.1007/s11336-014-9435-8

Orcutt, G. H., \& Irwin, J. O. (1948). A Study of the Autoregressive Nature of the Time Series Used for Tinbergen's Model of the Economic System of the United States, 1919-1932. Journal of the Royal Statistical Society. Series B (Methodological), 10(1), 1-53.

Orzek, J. H., Arnold, M., \& Voelkle, M. C. (2022). Striving for sparsity: On exact and approximate solutions in regularized structural equation models. [Manuscript submitted for publication].

Orzek, J. H., \& Voelkle, M. C. (in press). Regularized continuous time structural equation models: A network perspective. Psychological Methods.

Ou, L., Hunter, M. D., \& Chow, S.-M. (2019). What's for dynr: A package for linear and nonlinear dynamic modeling in R. The R journal, 11(1), 91.

Oud, J. H. L., Voelkle, M. C., \& Driver, C. C. (2018). Firstand Higher-Order Continuous Time Models for Arbitrary N Using SEM. In K. van Montfort, J. H. Oud, \& M. C. Voelkle (Eds.), Continuous Time Modeling in the Behavioral and Related Sciences (pp. 1-26). Springer International Publishing. https: //doi.org/10.1007/978-3-319-77219-6_1

Park, J. J., Chow, S.-M., Fisher, Z. F., \& Molenaar, P. C. M. (2020). Affect and personality: Ramifications of modeling (non-)directionality in dynamic network models. European Journal of Psychological Assessment, 36, 1009-1023. https:// doi .org/10 .1027/ 1015-5759/a000612

Park, T., \& Casella, G. (2008). The Bayesian Lasso. Journal of the American Statistical Association, 103(482), 681-686. https : // doi . org / 10 . 1198/ 016214508000000337

Pearl, J. (2009). Causal inference in statistics: An overview. Statistics Surveys, 3, 96-146. https://doi.org/10. 1214/09-SS057

Rothwell, P. M. (2006). Factors That Can Affect the External Validity of Randomised Controlled Trials. PLOS Clinical Trials, 1(1), e9. https://doi.org/10.1371/ journal.pctr.0010009

Rubin, D. B. (2005). Causal Inference Using Potential Outcomes. Journal of the American Statistical Association, 100(469), 322-331. https://doi.org/10. 1198/016214504000001880 _eprint: https://doi.org/10.1198/016214504000001880.

Ruissen, G. R., Zumbo, B. D., Rhodes, R. E., Puterman, E., \& Beauchamp, M. R. (2021). Analysis of dynamic psychological processes to understand and promote physical activity behaviour using intensive longitudinal methods: A primer. Health Psychology Review, 1-34.
Ryan, O., \& Hamaker, E. L. (2022). Time to intervene: A continuous-time approach to network analysis and centrality. Psychometrika, 87(1), 214-252.

Schuurman, N. K., \& Hamaker, E. L. (2019). Measurement error and person-specific reliability in multilevel autoregressive modeling. Psychological methods, 24(1), 70.

Schuurman, N. K., Houtveen, J. H., \& Hamaker, E. L. (2015). Incorporating measurement error in $\mathrm{n}=1$ psychological autoregressive modeling. Frontiers in Psychology, 0. https://doi.org/10.3389/fpsyg.2015. 01038

Shojaie, A., \& Fox, E. B. (2022). Granger Causality: A Review and Recent Advances. Annual Review of Statistics and Its Application, 9(1), 289-319. https: // doi . org / 10 . 1146/ annurev - statistics - 040120 010930

_eprint: https://doi.org/10.1146/annurev-statistics040120-010930.

Sims, C. A. (2010). Continuous and discrete time models. In Macroeconometrics and Time Series Analysis (pp. 60-67). Springer.

Spirtes, P., Glymour, C. N., Scheines, R., \& Heckerman, D. (2000). Causation, prediction, and search. MIT press.

Staudenmayer, J., \& Buonaccorsi, J. P. (2005). Measurement Error in Linear Autoregressive Models. Journal of the American Statistical Association, 100(471), 841-852. https : / / doi . org / 10 . 1198/ 016214504000001871 _eprint: https://doi.org/10.1198/016214504000001871.

Streeter, A. J., Lin, N. X., Crathorne, L., Haasova, M., Hyde, C., Melzer, D., \& Henley, W. E. (2017). Adjusting for unmeasured confounding in nonrandomized longitudinal studies: A methodological review. Journal of Clinical Epidemiology, 87, 23-34. https: //doi.org/10.1016/j.jclinepi.2017.04.022

Tibshirani, R. (1996). Regression shrinkage and selection via the lasso. Journal of the Royal Statistical Society: Series B (Methodological), 58(1), 267-288.

Timmons, A. C., \& Preacher, K. J. (2015). The Importance of Temporal Design: How Do Measurement Intervals Affect the Accuracy and Efficiency of Parameter Estimates in Longitudinal Research? Multivariate Behavioral Research, 50(1), 41-55. https://doi.org/10.1080/00273171.2014.961056 _eprint: https://doi.org/10.1080/00273171.2014.961056.

Voelkle, M. C., Gische, C., Driver, C. C., \& Lindenberger, U. (2019). The Role of Time in the Quest for Understanding Psychological Mechanisms. Multivariate Behavioral Research, 1-24. https://doi.org/10. 1080/00273171.2018.1496813 
Voelkle, M. C., \& Oud, J. H. L. (2013). Continuous time modelling with individually varying time intervals for oscillating and non-oscillating processes. British Journal of Mathematical and Statistical Psychology, 66(1), 103-126. https ://doi .org/10.1111/j. 2044-8317.2012.02043.x

Voelkle, M. C., \& Oud, J. H. (2015). Relating latent change score and continuous time models. Structural Equation Modeling: A Multidisciplinary Journal, 22(3), 366-381.

Voelkle, M. C., Oud, J. H., Davidov, E., \& Schmidt, P. (2012). An SEM approach to continuous time modeling of panel data: Relating authoritarianism and anomia. Psychological methods, 17(2), 176.
Wiener, N. (1956). The theory of prediction. Modern mathematics for engineers.

Yuen, T. P., Wong, H., \& Yiu, K. F. C. (2018). On constrained estimation of graphical time series models. Computational Statistics $\mathcal{E}$ Data Analysis, 124, 27-52. https://doi.org/10.1016/j.csda.2018.01.019

Zyphur, M. J., Allison, P. D., Tay, L., Voelkle, M. C., Preacher, K. J., Zhang, Z., Hamaker, E. L., Shamsollahi, A., Pierides, D. C., Koval, P., \& Diener, E. (2020). From Data to Causes I: Building A General Cross-Lagged Panel Model (GCLM). Organizational Research Methods, 23(4), 651-687. https: //doi.org/10.1177/1094428119847278 